\title{
Examining the Frequency of the $J A K 2$ (V617F) Mutation in Patients with Myeloproliferative Diseases in North Eastern Iran and the Effect of Treatment Intervention
}

\author{
Mojila Nasseri $^{\# 1,3}$, Fatemeh Keyfi ${ }^{\# 2,3}$, Raheleh Rahbarian ${ }^{1}$, \\ Majid Rajabian ${ }^{4}$, Mohammad Reza Abbaszadegan*3,5
}

\begin{abstract}
Background: Janus kinase 2 (JAK2) is a tyrosine kinase located in the cytoplasm that plays a critical role in the signal transduction of cytokines and growth hormones. The conversion of valine to phenylalanine at the polypeptide position 617 results in the JAK2 (V617F) mutation, which often found in patients with myeloproliferative neoplasms (MPNs). As a result of this mutation, $J A K 2$ is constitutively activated leading to uncontrolled cell growth. The present study aimed to investigate the frequency and relationship of the JAK2 (V617F) mutation in a population of patients with MPNs in Iran.

Methods: A total of 213 patients with myeloproliferative diseases (MPDs), were included in the study. Real-time PCR was used to detect the presence of the JAK2 (V617F) mutation in the genomic DNA isolated from patient peripheral blood samples.

Results: Of the 213 patients with MPDs, approximately 60 (28\%) patients were positive for the JAK2 (V617F) mutation. Polycythemia Vera (PV, 42.11\%) was the most common MPD, followed by Essential Thrombocythemia (ET, 29.82\%), Primary Myelofibrosis (MF, 12.28\%), and Chronic Myeloid Leukemia (CML, 10.5\%). A significant relationship between all types of MPDs and the clinical course $(p<$ 0.05) was observed. The relationship between age and gender among all types of MPD disease was not significant $(p>0.05)$.

Conclusions: Of the examined cohort in North Eastern Iran, 28\% of the patients with MPNs were found to have the JAK2 (V617F) mutation which determining the presence of the JAK2 (V617F) mutation helps to decide the correct form of treatment.
\end{abstract}

Keywords: AK2 (V617F), JAK-STAT pathway, MPD Real-Time PCR, Treatment.

\section{Introduction}

Myeloproliferative diseases (MPDs) are clonal stem cell disorders characterized by the presence of leukocytosis, thrombocytosis, erythrocytosis, an enlarged spleen, and multicellular bone marrow. In MPD, stem cells do not respond to the control mechanisms of hematopoietic cells. In vitro examination of stem cells has shown that cells cultured in serum without exogenous cytokines, cause continue to grow and develop clonal properties. This technique has become a standard

1: Department of Biochemistry, Payamenoor University, Tehran, Inan.

2: Department of Medical Laboratory Sciences, Varastegan Institute for Medical Sciences, Mashhad, Iran.

3: Pardis Clinical and Genetics Laboratory, Mashhad, Iran.

4: Department of biology, Payamenoor Univensity, Tehran, Iran.

5: Division of Human Genetics, Immunology Research Center, Avicenna Research Institute, MUMS, Mashhad, Iran.

\#These authors equally contributed to this study as first authors.

*Corresponding author: Mohammad Reza Abbaszadegan; Tel: +9851 37112343; E-mail: abbaszadeganmr@mums.ac.ir.

Received: 25 Aug, 2019; Accepted: 2 Sep, 2019 
method in diagnosis of MPD. Mutations in the $J A K 2$, CALR, and MPL genes are commonly found in individuals with MPDs, of which the mutation in the JAK 2 gene is the most significant (1). The different MPDs include Polycythemia Vera (PV), Essential Thrombocytosis (ET), Chronic Idiopathic Myelofibrosis (IMF), and Chronic Myelogenous Leukemia (CML), which are classified based on the dominant cell type with abnormally high growth and proliferation. The discovery of the presence of the JAK2 (V617F) mutation in MPNs was an important advancement in the understanding MPD pathogenesis (2).

The JAK2 gene is located on chromosome 9 at position (P249) and is a member of the JAK family of tyrosine kinases. The JAK2 tyrosine kinase is located within the cytoplasm and holds an important role in the signal transduction of cytokines by growth hormones. The JAK2 (V617F) mutation is an acquired, somatic mutation caused by a guanine to thymine ( $\mathrm{G}$ to $\mathrm{T}$ ) transversion at nucleotide 1849 in exon 14, which results in the conversion of the amino acid valine to phenylalanine at polypeptide chain position 617 (V617F). The mutation is located in the second pseudo-kinase $\mathrm{JH} 2$ from $J A K 2$, involved in limiting kinase activity. These changes result in constitutive JAK2 activation, increasing sensitivity to erythropoietin (EPO) and enabling growth independent of growth factors (3). Receptors for growth hormone, prolactin, EPO, and cytokines are devoid of intrinsic tyrosine kinase activity. Cytokine receptors have associated cytosolic tyrosine kinases, such as JAK, bound to their cytoplasmic region. The binding of cytokines to the extracellular region of their respective receptors results in receptor dimerization, facilitating the trans-phosphorylation of the associated JAKs. JAK dimers phosphorylate the subunits of other cytokine receptors in the tyrosine roots. These phosphorylation units then act as identification sites for binding other signaling proteins to the interaction regions of Src Homology Type 2 (SH2). JAK dimers in turn lead to the phosphorylation and dimerization of monomer proteins of Signal Transducers and Activators of Transcription (STAT). The activated STAT dimers travel to the nucleus and stimulate gene expression. As a result of the JAK2 (V617F) mutation, $J A K 2$ is permanently activated leading to uncontrolled cell growth in the absence of growth hormones (4،5). The SOSC and CBL proteins normally degrade JAK2 via protein ubiquitination preventing continued signal transduction. The $J A K 2$ (V617F) mutation makes the JAK2 protein resistant to degradation by the SOSC and CBL proteins. The use of JAK inhibitors disables the JAK-STAT pathway, preventing gene transcription and expression (2, 6-8).The JAK2 (V617F) mutation is commonly found in patients with MPD, negative for the Breakpoint Cluster Region-Abelson Leukemia (BCR-ABL) mutation (9). Due to the lack of research evaluating the presence and role of the $J A K 2(\mathrm{~V} 617 \mathrm{~F})$ mutation in the population of North Eastern Iran and its importance in the diagnosis of MPDs, the present study was conducted to investigate this relationship and the impact of treatment intervention.

\section{Materials and methods}

This study was performed in the North East of Iran. A total of 213 patients referred to Pardis Laboratory of Mashhad from 2009-20017 with symptoms of MPD were included in the study. Patients were screened for the JAK2 (V617F) mutation. Questionnaire forms were given to patients determined to have the JAK2 (V617F) mutation and diagnosed by a physician with MPD. In this questionnaire, the history, symptoms of disease, medication, bone marrow biopsies and tests performed were evaluated. Peripheral whole blood samples were isolated from patients in a nonfasting state, a total of $3 \mathrm{~mL}$ were collected. Blood was collected in sterile tubes containing EDTA anticoagulant. According to the protocol kit, the High Pure PCR Template Preparation Kit Germany (from ROCHE) with Cat \# 11796828001 was extracted using proteinase method. DNA was extracted from $200 \mu$ lof blood and dissolved in $50 \mu$ lElution Buffer. The presence of the JAK2 (V617F) mutation was evaluated by Real Time PCR method using the Novin Gene kit. The detection sensitivity of this kit is equivalent to $0.1 \%$ or one per thousand. For example, if only one out of a thousand white blood cells have the JAK2 (V617F) mutation, it will be diagnosed in $95 \%$ of cases. In this experiment, the patient DNA samples were examined with several controls including 
positive and negative controls for the JAK2 (V617F) mutation. The results were evaluated with the help of Applied Biosystems (ABI) Step One Plus Machine Real-Time PCR software V 7.7. Following the confirmation of the JAK2 (V617F) mutation, the patients began their course of treatment. Six months after starting treatment, we contacted patients and learned about the effect of the administered drug on improving the disease. Data analysis was performed using SPSS software.

Table 1. Characteristics and clinical course of the 60 patients with the JAK2 (V617F) mutation.

\begin{tabular}{lcc}
\cline { 2 - 2 } & Number & Percent \\
\hline Sex: male/female & $31 / 29$ & $51.7 / 48.3$ \\
Age: mean (Range) & $58.35(20-80)$ & \\
Diagnosis: & & \\
PV/ET/MF/CML/AML/ET or MF & $24 / 17 / 7 / 6 / 1 / 2$ & $42 / 29 / 12 / 10 / 1 / 3$ \\
Treatments: & & \\
Phlebotomy & 2 & 3.3 \\
Hydroxyurea & 23 & 46.7 \\
Drug/bone marrow transplant & 1 & 1.7 \\
Drug/phlebotomy & 3 & 5 \\
Drug/chemotherapy & 1 & 1.7 \\
Leech therapy & 1 & 1.7 \\
chemotherapy & 2 & 3.3 \\
History available: +/- & $2 / 35$ & $3.3 / 58.3$ \\
Condition available: died/under treatment & $9 / 31$ & $15 / 51.7$ \\
\hline
\end{tabular}

*PV: Polycythemia Vera, ET: Essential Thrombocytosis, MF: Idiopathic Myelofibrosis, CML: Chronic Myelogenous Leukemia, AML: Acute Myelocytic Leukemia.

\section{Results}

In this study, 213 patients diagnosed with a form of MPD in the North East of Iran were examined. Of the patients included in this study, $60(28 \%)$ were found to have the JAK2 (V617F) mutation. Data analysis was conducted in two descriptive and analytic sections. According to the descriptive analysis (Table 1), the mean age of patients was 58 years with the overall age ranging from 20-88 years old. Of the patients with the JAK2 (V617F) mutation, 29 (48.3\%) were women and $31(51.7 \%)$ were men. Among the different types of MPD, 24 patients $(42.11 \%)$ had Polycythemia Vera (PV), 17 patients $(29.82 \%)$ were diagnosed with Essential Thrombocythemia (ET), 7 patients (12.28\%) with Primary Myelofibrosis (PMF), and 6 patients (10.5\%) with Chronic Myeloid Leukemia (CML). Only $1(1.75 \%)$ of the patients with the JAK2 (V617F) mutation was diagnosed with Acute Myelocytic Leukemia (AML). It should be noted that $2(3.5 \%)$ of the 60 patients with the JAK2 (V617F) mutation had problems due to insufficient patient history of ET/MF diagnosis, and further examinations and testing was required.

\section{Discussion}

Various studies have been conducted within Iran and abroad examining the role of the JAK2 (V617F) mutation in patients with MPDs. Bagheri et al. examined the presence of the tyrosine kinase $J A K 2$ (V617F) mutation in patients diagnosed with BCR/ABL-negative chronic MPD in the province of West Azerbaijan. In this study, the frequency of the JAK2 (V617F) mutation was determined to be $76.74 \%$, indicating that this mutation is present in the majority of Iranian West Azerbaijani patients with BCR/ABL-negative chronic MPDs. Identifying the presence of the JAK2 (V617F) mutation is an important aspect in determining the course of treatment for patients with MPD. Considering that the prevalence of this mutation in the North Eastern population of Iran has not been evaluated and that the presence of the JAK2 (V617F) mutation can influence treatment, we explored this issue here (10).

Previous research by Langabeer et al. indicated that the JAK2 (V617F) mutation was the major diagnostic criterion for MPNs, such as PV, ET, and PMF. Moreover, this mutation has also been 
observed in other myeloid malignancies such as acute myelogenous leukemia, myelodysplastic syndrome and thrombocytopenia (11).

Bhagwat et al. examined the effect of targeting $J A K 2$ via JAK inhibitors and JAK2 deletions on the disease progression of MPN. Their findings revealed that blocking or eliminating JAK2 function significantly reduced disease burden. Their findings suggest that identifying the presence of the JAK2 (V617F) mutation was not only of diagnostic importance, but also a major target involved in the successful treatment of chronic MPNs via JAK/STAT pathway inhibitors (3). The results of this study were consistent previous studies, indicating that the different forms of MPD positive for the JAK2 (V617F) mutation was most frequently found in PV, followed by ET and PMF, and least commonly CML. Our findings showed that the best treatment method for the patients of was the use of hydroxyurea and most of them were satisfied with the drug and immediately began the treatment process after the disease diagnosis and the disease was controlled. In addition to medication, $5 \%$ of patients received phlebotomy every two months. Due to hydroxyurea sensitivity, one of the patients receiving treatment used the alternative traditional medicine method of leech therapy 3 times a year. The patient indicated an improvement of symptoms associated with PV. In this study, only $3 \%$ of the patients had a history of blood diseases. In the remaining patients there was no history of blood disorders in their families or relatives, which indicated the acquisition of the JAK2 (V617F) mutation. Of the patients in this study, $15 \%$ with the $J A K 2$ (V617F) mutation died and the remaining patients continued with treatment. The relationship between various types of MPDs and patient status was investigated.

\section{References}

1. Kim A, Strober B. Janus Kinase Inhibitors. Biologic and Systemic Agents in Dermatology: Springer; 2018. P. 187-98.

2.Percy MJ, Zhao Q, Flores A, Harrison C, Lappin TR, Maxwell PH, et. al. A family with erythrocytosis establishes a role for prolyl hydroxylase domain protein 2 in oxygen homeostasis. Proc Natl Acad Sci U S A. 2006;103(3):654-9.
Given that the patients treated in a variety of MPDs were more than the people who died, it can be concluded that there was a significant relationship $(p<0.05)$ between the different forms of MPD and the status of the patients. Consequently, treatment had a significant role in the survival of patients with the JAK2 (V617F) mutation. The relationship between gender and age with respect to the different MPDs was examined. No statistically significant relationship was found $(p>$ 0.05 ). This suggests that the presence of the JAK2 (V617F) mutation in patients did not depend on gender and age, and the mutation was completely acquired. The JAK2 (V617F) mutation has been shown to be an accurate diagnostic indicator for MPDs in people found to be BCR/ABL-negative. This information was confirmed by the Clinical Advisory Committee for Review of the World Health Organization's classification for myeloid neoplasms and its approval is recommended by the World Health Organization (WHO) (12).

Due to the frequency of the JAK2 (V617F) mutation $(28 \%)$ in various MPDs in patients from North East Iran and the significant relationship between MPDs and patient status, we conclude that the presence of the JAK2 (M617F) mutation is an important diagnostic indictor for patients with PV, ET, PMF, and CML. Additionally, identifying the presence of the JAK2 (V617F) mutation is not only diagnostically important, but also important in determining the treatment and treatment outcome of MPD using JAK/STAT pathway inhibitors $(3,13)$.

\section{Acknowledgment}

We appreciate all patients who participated in this study.

3. Bhagwat N, Koppikar P, Keller M, Marubayashi S, Shank K, Rampal R, et al. Improved targeting of $J A K 2$ leads to increased therapeutic efficacy in myeloproliferative neoplasms. Blood. 2014;123(13):2075-83.

4. Ghafari H, Karimzadeh P, Chahardouli B, Alimoghdam K, Ghavamzadeh A, Dargahi H, et al. Evaluation of JAK2V617F mutation prevalence in Myeloproliferative Neoplasm by 
AS-RT-PCR. Journal of Payavard Salamat.2009;3(2):94-101.

5. Hubbard SR. Mechanistic insights into Regulation of JAK2 Tyrosine Kinase. Front Endocrinol (Lausanne). 2018;8:361.

6. Ayatollahi H, Sadeghian MH, Keramati MR, Karimiani EG, Jafarian AH, Shirdel A, et al. JAK2 V617F Mutation in Adult T Cell LeukemiaLymphoma. Indian J Hematol Blood Transfus. 2016;32(4):437-441.

7. Saharinen P, Takaluoma K, Silvennoinen O. Regulation of the Jak2 tyrosine kinase by its pseudokinase domain. Mol Cell Biol. 2000; 20(10):3387-3395.

8. Williams JJ, Munro K, Palmer TM. Role of Ubiquitylation in Controlling Suppressor of Cytokine Signalling 3(SOCS3) Function and Expression. Cells. 2014;3(2):546-562.

9. Ebid GT, Ghareeb M, Salaheldin O, Kamel MM. Prevalence of the frequency of JAK2 (V617F) mutation in different myeloproliferative disorders in
Egyptian patients. Int J Clin Exp Pathol. 2015;8(9):11555-11559.

10. Bagheri M, Abdirad I, Maleki D, Eyshi A, Valizadeh N. Acquired mutation of the tyrosine kinase $J A K 2 \mathrm{~V} 617 \mathrm{~F}$ in the ABL-BCR-negative chronic myeloproliferative diseases in a population of west Azerbaijan province, Iran. Journal of Isfahan Medical School. 2018;35(459):1785-1791. 11. Langabeer SE. The $J A K 2$ V617F mutation in isolated neutropenia. EXCLI J. 2018;17:1-2.

12. Barosi G, Mesa R, Thiele J, Cervantes F, Campbell P, Verstovsek S, et al. Proposed criteria for the diagnosis of post-polycythemia vera and post-essential thrombocythemiamyelofibrosis: a consensus statement from the International Working Group for Myelofibrosis Research and Treatment. Leukemia. 2008;22(2):437-8.

13. Neet K, Hunter T. Vertebrate non-receptor protein-tyrosine kinase families. Genes Cells. 1996;1(2):147-69. 